\title{
Cores of Convex and Strictly Convex Games*
}

\author{
JuLIO GONZÁLEZ-DÍAZ ${ }^{\dagger}$ \\ Kellogg School of Management (CMS-EMS), Northwestern University \\ and \\ Research Group in Economic Analysis, University of Vigo \\ Estela SÁnchez-Rodríguez \\ Department of Statistics and Operations Research, University of Vigo \\ Published in Games and Economic Behavior (2008) 62, 100-105 \\ Published version available at http://www.sciencedirect.com \\ DOI $10.1016 /$ j.geb.2007.03.003
}

\begin{abstract}
We follow the path initiated in Shapley (1971) and study the geometry of the core of convex and strictly convex games. We define what we call face games and use them to study the combinatorial complexity of the core of a strictly convex game. Remarkably, we present a picture that summarizes our results with the aid of Pascal's triangle.
\end{abstract}

JEL classification: C71.

Keywords: cooperative TU games, convex games, combinatorial complexity, Pascal's triangle.

\section{Preliminaries}

A cooperative $n$-player game with transferable utility, shortly, a TU game, is a pair $(N, v)$, where $N$ is a finite set and $v: 2^{N} \rightarrow \mathbb{R}$ is a function assigning, to each coalition $S \in 2^{N}$, its worth $v(S)$; by convention $v(\emptyset):=0$. Let $G^{n}$ be the set of $n$-player games. Given $S \subseteq N$, let $|S|$ be the number of players in $S$. For the sake of notation, we denote $\{i\}$ by $i$.

Let $(N, v) \in G^{n}$. The core (Gillies, 1953), is defined by $C(N, v):=\left\{x \in \mathbb{R}^{n}: \sum_{i \in N} x_{i}=\right.$ $v(N)$ and, for each $\left.S \subseteq N, \sum_{i \in S} x_{i} \geq v(S)\right\}$. Let $B G^{n}$ be the set of $n$-player games with nonempty core. We say $C(N, v)$ is full dimensional if it has dimension $n-1$.

Let $S \subseteq N$ and let $\Pi(S)$ be the set of orderings (permutations) of the elements in $S$. For each $\sigma_{S} \in \Pi(S)$ and each $i \in S, \sigma_{S}(i)$ denotes $i$ 's position. We denote $\sigma_{N}$ by $\sigma$. For each $i \in N$ and each $\sigma \in \Pi(N)$, let $P_{\sigma}(i):=\{j \in N: \sigma(j)<\sigma(i)\}$ be the set of predecessors of $i$ with respect to $\sigma$. Let $(N, v) \in G^{n}$ and $\sigma \in \Pi(N)$. The marginal vector associated with $(N, v)$ and $\sigma, m^{\sigma}(N, v)$, is defined, for each $i \in N$, by $m_{i}^{\sigma}(N, v):=v\left(P_{\sigma}(i) \cup i\right)-v\left(P_{\sigma}(i)\right)$.

\footnotetext{
*Acknowledgements. The authors are grateful to Miguel A. Meléndez-Jiménez and two anonymous referees for helpful comments and acknowledge the financial support of the Spanish Ministry for Science and Education through projects SEJ2005-07637-C02-02 and from the Xunta de Galicia under project PGIDIT06PXIC207038PN. Julio González Díaz also acknowledges support from projects SEJ2005-01481C02-02 and BEC2003-01132.

${ }^{\dagger}$ Corresponding author: Kellogg School of Management (CMS-EMS), 5100 Leverone Hall, 2001 Sheridan Road. Evanston, IL 60208-2014. United States. email: julio@northwestern.edu
} 
A game $(N, v)$ is convex if, for each $i \in N$ and each $S$ and $T$ such that $S \subseteq T \subseteq N \backslash\{i\}$, $v(S \cup i)-v(S) \leq v(T \cup i)-v(T)$. Let $C G^{n}$ be the set of $n$-player convex games. A game $(N, v)$ is strictly convex if, for each $i \in N$ and each $S$ and $T$ such that $S \subsetneq T \subseteq N \backslash\{i\}$, $v(S \cup i)-v(S)<v(T \cup i)-v(T)$.

A (convex) polytope $P$ is the convex hull of a finite set of points. A polytope $P$ is an $m$-polytope if its dimension is $m$. A hyperplane $H$ is a supporting hyperplane for $P$ if $H \cap P \neq \emptyset$ and the halfspace below $H$ contains $P$. A face of a polytope $P$ is defined as (i) $P$ itself, (ii) the empty set, or (iii) the intersection of $P$ with some supporting hyperplane; faces of dimension $m$ are called $m$-faces (with the convention that $\operatorname{dim}(\emptyset)=-1$ ). The 0 -faces, 1 -faces, and $(m-1)$-faces of an $m$-polytope $P$ are respectively its vertices, edges, and facets. Let $\mathcal{F}(P)$ denote the set of all faces of $P$.

\section{Core Complexity and Pascal's Triangle}

We discuss now the geometry of the cores of convex and strictly convex games. In our exposition we mainly use the terminology in Shaplev (1971) and, also, some of the results included there. Let $(N, v) \in B G^{n}$. For each $\emptyset \neq T \subseteq N$, let $H_{T}$ be the hyperplane $H_{T}:=\left\{x \in \mathbb{R}^{n}: \sum_{i \in T} x_{i}=v(T)\right\}$ and let $F_{T}:=C(N, v) \cap H_{N \backslash T}$. Clearly, $F_{\emptyset}=C(N, v)$; also, let $F_{N}:=C(N, v) 1$ Shapley (1971) and Ichiishi (1981) showed that a game is convex if and only if the vertices of the core are the marginal vectors, i.e., $C(N, v)=\operatorname{co}\left\{m^{\sigma}(N, v)\right.$ : $\sigma \in \Pi(N)\}$, where $\operatorname{co}(A)$ denotes the convex hull of $A$. Thus, for convex games, each $F_{T}$ is a nonempty face of $C(N, v)$ and we refer to $F_{T}$ as a $T$-face of $C(N, v)$. By definition, in each allocation in $F_{T}$, coalition $T$ receives $v(N)-v(N \backslash T)$. Clearly, for each $\emptyset \neq T \subsetneq N$, since both $F_{T}$ and $F_{N \backslash T}$ lie in $H_{N}$, they are parallel to each other. Now we define, for each coalition $T \subseteq N$, a game $\left(N, v_{F_{T}}\right)$ that is closely related to $F_{T}$.

The idea of the definition is the following. Given a convex game $(N, v)$ and a face $F_{T}$ of its core, if we restrict attention to $C(N, v)$, then the allocations in $F_{T}$ are the best ones for $T$ and the worst ones for $N \backslash T$. Coalition $T$ always receives $v(N)-v(N \backslash T)$ and coalition $N \backslash T$ gets $v(N \backslash T)$. Yet, there is still freedom for $v(N)-v(N \backslash T)$ to be shared among the players in $T$ and for $v(N \backslash T)$ to be shared among the ones in $N \backslash T$. Next definition captures this idea.

Definition 1. Let $(N, v) \in B G^{n}$ and $T \subseteq N$. The $T$-face game $\left(N, v_{F_{T}}\right)$ is defined, for each $S \subseteq N$, by $v_{F_{T}}(S):=v((S \cap T) \cup(N \backslash T))-v(N \backslash T)+v(S \cap(N \backslash T))$.

Note that, if $T=\emptyset$ or $T=N$, then $\left(N, v_{F_{T}}\right)=(N, v)$. Besides, if $S \cap T=\emptyset$, then $v_{F_{T}}(S)=v(S)$. If $\emptyset \neq T \subsetneq N$ then, in the game $\left(N, v_{F_{T}}\right)$, the worths of coalitions $T$ and $N \backslash T$ are $v_{F_{T}}(T)=v(N)-v(N \backslash T)$ and $v_{F_{T}}(N \backslash T)=v(N \backslash T)$, respectively. Moreover, if $(N, v) \in C G^{n}$, then $v_{F_{T}}(T)$ is also the maximum payoff that coalition $T$ can get in $C(N, v)$; similarly, $v_{F_{T}}(N \backslash T)$ is the minimum for $N \backslash T$.

Lemma 1. Let $(N, v) \in B G^{n}$ and $T \subseteq N$. Let $\sigma=\left(\sigma_{N \backslash T}, \sigma_{T}\right)$ and let $\bar{\sigma} \in \Pi(N)$ be such that it induces the orders $\sigma_{T}$ and $\sigma_{N \backslash T}$ in $T$ and $N \backslash T$, respectively. Then,

(i) $m^{\sigma}\left(N, v_{F_{T}}\right)=m^{\sigma}(N, v)$.

(ii) $m^{\sigma}\left(N, v_{F_{T}}\right)=m^{\bar{\sigma}}\left(N, v_{F_{T}}\right)$.

Moreover, if $(N, v) \in C G^{n}$ then,

\footnotetext{
[Shapley (1971) defines $F_{T}$ as $C(N, v) \cap H_{T}$. Although Shapley's definition might seem more natural, ours is more convenient for the exposition below.
} 
(iii) $\left(N, v_{F_{T}}\right) \in C G^{n}$.

(iv) $m^{\bar{\sigma}}(N, v) \in F_{T}$ if and only if $m^{\sigma}(N, v)=m^{\bar{\sigma}}(N, v)$. Hence, $F_{T}=\operatorname{co}\left\{m^{\sigma}(N, v): \sigma=\right.$ $\left.\left(\sigma_{N \backslash T}, \sigma_{T}\right)\right\}$.

Proof. Since $\left(N, v_{F_{\emptyset}}\right)=\left(N, v_{F_{N}}\right)=(N, v)$, the result is trivial for $T=\emptyset$ and $T=N$. Hence, let $\emptyset \neq T \subsetneq N$.

(i) Let $\sigma=\left(\sigma_{N \backslash T}, \sigma_{T}\right)$. We show that, for each $i \in N, m_{i}^{\sigma}(N, v)=m_{i}^{\sigma}\left(N, v_{F_{T}}\right)$. Suppose that $i \in N \backslash T$. Since $P_{\sigma}(i) \subset P_{\sigma}(i) \cup i \subseteq N \backslash T$, then $v\left(P_{\sigma}(i) \cup i\right)=v_{F_{T}}\left(P_{\sigma}(i) \cup i\right)$ and $v\left(P_{\sigma}(i)\right)=v_{F_{T}}\left(P_{\sigma}(i)\right)$. Suppose that $i \in T$. In this case, $N \backslash T \subseteq P_{\sigma}(i)$ and it is easy to check that, again, $v_{F_{T}}\left(P_{\sigma}(i) \cup i\right)=v\left(P_{\sigma}(i) \cup i\right)$ and $v_{F_{T}}\left(P_{\sigma}(i)\right)=v\left(P_{\sigma}(i)\right)$.

(ii) If $\bar{\sigma}=\left(\sigma_{N \backslash T}, \sigma_{T}\right)$ the result is trivial. Let $\bar{\sigma} \neq\left(\sigma_{N \backslash T}, \sigma_{T}\right)$. Then, $\bar{\sigma}$ can be written as $\left(\sigma_{R_{1}}, \sigma_{T_{1}}, \sigma_{R_{2}}, \sigma_{T_{2}}, \ldots, \sigma_{R_{p}}, \sigma_{T_{q}}\right)$, where $T_{1}, \ldots, T_{q} \subset T, R_{1}, \ldots, R_{p} \subset N \backslash T$, and $T_{1}$ and $R_{2}$ are nonempty. Let $\sigma^{*}:=\left(\sigma_{R_{1}}, \sigma_{R_{2}}, \sigma_{T_{1}}, \sigma_{T_{2}}, \ldots, \sigma_{R_{p}}, \sigma_{T_{q}}\right)$, i.e., $R_{2}$ and $T_{1}$ are swapped. We show that $m^{\bar{\sigma}}\left(N, v_{F_{T}}\right)=m^{\sigma^{*}}\left(N, v_{F_{T}}\right)$. Once the latter is proved, we get, after a finite number of swaps, that $m^{\bar{\sigma}}\left(N, v_{F_{T}}\right)=m^{\sigma}\left(N, v_{F_{T}}\right)$. Clearly, the marginal vectors associated with $\bar{\sigma}$ and $\sigma^{*}$ can only differ for the players in $T_{1}$ or $R_{2}$. We distinguish two cases. Case 1: $i \in T_{1}$. Clearly, $P_{\bar{\sigma}}(i)=R_{1} \cup P_{\sigma_{T_{1}}}(i)$ and $P_{\sigma^{*}}(i)=R_{1} \cup R_{2} \cup P_{\sigma_{T_{1}}}(i)$. By the definition of $v_{F_{T}}, v_{F_{T}}\left(P_{\bar{\sigma}}(i) \cup i\right)-v_{F_{T}}\left(P_{\bar{\sigma}}(i)\right)=v\left(P_{\sigma_{T_{1}}}(i) \cup(N \backslash T) \cup i\right)-v\left(P_{\sigma_{T_{1}}}(i) \cup(N \backslash T)\right)=$ $v_{F_{T}}\left(P_{\sigma^{*}}(i) \cup i\right)-v_{F_{T}}\left(P_{\sigma^{*}}(i)\right)$. Case 2: $i \in R_{2}$. Clearly, $P_{\bar{\sigma}}(i)=R_{1} \cup T_{1} \cup P_{\sigma_{R_{2}}}(i)$ and $P_{\sigma^{*}}(i)=R_{1} \cup P_{\sigma_{R_{2}}}(i)$. By the definition of $v_{F_{T}}, v_{F_{T}}\left(P_{\bar{\sigma}}(i) \cup i\right)-v_{F_{T}}\left(P_{\bar{\sigma}}(i)\right)=v\left(R_{1} \cup\right.$ $\left.P_{\sigma_{R_{2}}}(i) \cup i\right)-v\left(R_{1} \cup P_{\sigma_{R_{2}}}(i)\right)=v_{F_{T}}\left(P_{\sigma^{*}}(i) \cup i\right)-v_{F_{T}}\left(P_{\sigma^{*}}(i)\right)$.

(iii) We show that, for each $R \subseteq S \subseteq N \backslash i, v_{F_{T}}(R \cup i)-v_{F_{T}}(R) \leq v_{F_{T}}(S \cup i)-v_{F_{T}}(S)$. Suppose that $i \in N \backslash T$. Since $v_{F_{T}}(S \cup i)-v_{F_{T}}(S)=v((S \cup i) \cap(N \backslash T))-v(S \cap(N \backslash T))$, $v_{F_{T}}(R \cup i)-v_{F_{T}}(R)=v((R \cup i) \cap(N \backslash T))-v(R \cap(N \backslash T))$, and $(N, v)$ is convex, the desired inequality holds. Suppose that $i \in T$. Since $v_{F_{T}}(S \cup i)-v_{F_{T}}(S)=v((S \cap T) \cup(N \backslash T) \cup i)-$ $v((S \cap T) \cup(N \backslash T)), v_{F_{T}}(R \cup i)-v_{F_{T}}(R)=v((R \cap T) \cup(N \backslash T) \cup i)-v((R \cap T) \cup(N \backslash T))$, and $(N, v)$ is convex, the desired inequality holds.

(iv) Since $v \in C G^{n}, m^{\sigma}(N, v) \in F_{T}$ and the necessity is trivial. We prove the sufficiency. Since $m^{\bar{\sigma}}(N, v) \in F_{T}$, then $\sum_{i \in T} m_{i}^{\bar{\sigma}}=v(N)-v(N \backslash T)=\sum_{i \in T} m_{i}^{\sigma}$. By convexity, for each $i \in T, m_{i}^{\bar{\sigma}}(N, v) \leq m_{i}^{\sigma}(N, v)$ and, since $\sum_{i \in T} m_{i}^{\bar{\sigma}}=\sum_{i \in T} m_{i}^{\sigma}$, we have that, for each $i \in T$, $m_{i}^{\bar{\sigma}}(N, v)=m_{i}^{\sigma}(N, v)$. Similarly, for each $i \in N \backslash T, m_{i}^{\bar{\sigma}}(N, v)=m_{i}^{\sigma}(N, v)$.

Proposition 1. Let $(N, v) \in C G^{n}$ and $T \subseteq N$. Then, $C\left(N, v_{F_{T}}\right)=F_{T}$. Therefore, $C(N, v)=\operatorname{co}\left\{C\left(N, v_{F_{T}}\right): \emptyset \neq T \subsetneq N\right\}$.

Proof. The equality $C\left(N, v_{F_{T}}\right)=F_{T}$ is trivial for $T=\emptyset$ and $T=N$. Let $\emptyset \neq T \subsetneq N$. By Lemma 1 (i), for each $\sigma \in \Pi(N), m^{\sigma}\left(N, v_{F_{T}}\right)$ is a vertex of $C\left(N, v_{F_{T}}\right)$ and $C\left(N, v_{F_{T}}\right)=$ $\operatorname{co}\left\{m^{\sigma}\left(N, v_{F_{T}}\right): \sigma \in \Pi(N)\right\}$. Now, by Lemma 1 (ii) and (iii), $C\left(N, v_{F_{T}}\right) \subseteq F_{T}$ and, by Lemma 1 (ii) and (iv), $F_{T} \subseteq C\left(N, v_{F_{T}}\right)$.

The following result is a compilation of different results in Shapley (1971).

Lemma 2. Let $(N, v)$ be a strictly convex game. Then,

(i) $m^{\sigma}(N, v)=m^{\bar{\sigma}}(N, v)$ if and only if $\sigma=\bar{\sigma}$. Hence, $C(N, v)$ has $n$ ! vertices.

(ii) $C(N, v)$ is full dimensional and has $2^{n}-2$ facets, one for each $\emptyset \neq T \subsetneq N$.

(iii) Let $\emptyset \neq T \subsetneq N$. Then, $m^{\sigma}(N, v) \in F_{T}$ if and only if $\sigma$ is of the form $\left(\sigma_{N \backslash T}, \sigma_{T}\right)$. 
Remark. From the previous result, for each strictly convex game and each $\emptyset \neq T \subsetneq N$, $F_{T}$ is a facet of $C(N, v)$, i.e., an $(n-2)$-polytope. Moreover, (i) and (iii) imply that $F_{T}$ has $|T| !(n-|T|)$ ! vertices and, hence, $\left(N, v_{F_{T}}\right)$ is not strictly convex. Recall that, for each $t \in\{0, \ldots, n\}$, the number of $t$-player coalitions is $\left(\begin{array}{l}n \\ t\end{array}\right)$. Hence, $\left(\begin{array}{l}n \\ t\end{array}\right)$ is also the number of faces of $C(N, v)$ that are associated with a coalition of size $t$.

Now, we introduce one more concept from Shapley (1971). Let $\mathcal{P}=\left\{N_{1}, \ldots, N_{p}\right\}$ be a partition of $N$, with $p \geq 2$. The game $(N, v)$ is decomposable with respect to $\mathcal{P}$ if, for each $S \subseteq N, v(S)=v\left(S \cap N_{1}\right)+\ldots+v\left(S \cap N_{p}\right)$. That is, $v$ is the addition of $p$ smaller games; each of them is referred to as a component. The following result is also a compilation of different results in Shapley (1971).

Lemma 3. (i) A strictly convex game is indecomposable.

(ii) A decomposable game is convex if and only if each component is convex.

(iii) The core of a decomposable convex game is the cartesian product of the cores of the components of any decomposition.

Proposition 2. Let $(N, v) \in B G^{n}$ and $\emptyset \neq T \subsetneq N$. Then, the game $\left(N, v_{F_{T}}\right)$ is decomposable with respect to $\mathcal{P}=\{T, N \backslash T\}$.

Proof. Let $S \subseteq N$. Then, $v_{F_{T}}(S \cap T)=v((S \cap T) \cup(N \backslash T))-v(N \backslash T)+v(\emptyset)$ and $v_{F_{T}}(S \cap$ $(N \backslash T))=v(N \backslash T)-v(N \backslash T)+v(S \cap(N \backslash T))$. Hence, $v_{F_{T}}(S \cap T)+v_{F_{T}}(S \cap(N \backslash T))=$ $v_{F_{T}}(S)$.

Let $\left(T, v^{T}\right)$ and $\left(N \backslash T, v^{N \backslash T}\right)$ denote the two components of the decomposition in Proposition 2. Next result is now completely straightforward.

Corollary 1. Let $(N, v)$ be a strictly convex game and $\emptyset \neq T \subsetneq N$. Then, $\left(T, v^{T}\right)$ and $\left(N \backslash T, v^{N \backslash T}\right)$ are strictly convex games such that $C\left(N, v_{F_{T}}\right)=C\left(T, v^{T}\right) \times C\left(N \backslash T, v^{N \backslash T}\right)$. The cores in the cartesian product have dimensions $|T|-1$ and $|N \backslash T|-1$, respectively.

We move now to core complexity. Two polytopes $P$ and $P^{\prime}$ are combinatorially equivalent if there is a one-to-one map $f: \mathcal{F}(P) \rightarrow \mathcal{F}\left(P^{\prime}\right)$ that is inclusion preserving, i.e., $F \subseteq F^{\prime}$ if and only if $f(F) \subseteq f\left(F^{\prime}\right)$. We define the combinatorial complexity of the core of a game as the number of different equivalence classes there are among its facets according to the above relation. Given a strictly convex game and a coalition $\emptyset \neq T \subsetneq N$, all the $|T|$-faces are combinatorially equivalent and, moreover, the faces $F_{T}$ and $F_{N \backslash T}$ are also combinatorially equivalent. Let $\lfloor\cdot\rfloor$ be the floor function, i.e., for each $r \in \mathbb{R},\lfloor r\rfloor$ denotes the largest integer not larger than $r$. The following corollaries are immediate from Lemma 2 and the remark below it.

Corollary 2. Let $(N, v)$ be a strictly convex game. Then, for each $t \in\{1, \ldots, n-1\}$, $C(N, v)$ has $2\left(\begin{array}{c}n \\ t\end{array}\right)$ combinatorially equivalent facets and each of them can be decomposed as the cartesian product of the cores of two strictly convex games with $t$ and $n-t$ players, respectively.

Corollary 3. Let $(N, v)$ and $(N, w)$ be two strictly convex games. Then, $C(N, v)$ and $C(N, w)$ are combinatorially equivalent. Indeed, given $t \in\{0, \ldots, n\}$, for each $T, S \subseteq N$ such that $|T|=|S|=t, C\left(N, v_{F_{T}}\right)$ and $C\left(N, w_{F_{S}}\right)$ are combinatorially equivalent.

Corollary 4. Let $(N, v)$ be a strictly convex game. Then, the combinatorial complexity of $C(N, v)$ is $\left\lfloor\frac{n}{2}\right\rfloor$. 
In Figure 1 we illustrate, with the aid of Pascal's triangle, the above corollaries. Given $n$, below $\left(\begin{array}{l}n \\ 0\end{array}\right)$ and $\left(\begin{array}{l}n \\ n\end{array}\right)$, we draw the $F_{\emptyset}$ and $F_{N}$ faces, i.e., $C(N, v)$. Then, for each $t \in$ $\{1, \ldots, n-1\}$, the polytope below $\left(\begin{array}{l}n \\ t\end{array}\right)$ represents one of the $\left(\begin{array}{l}n \\ t\end{array}\right)$ combinatorially equivalent $T$-facets (with $|T|=t$ ) of $C(N, v)$ and, since $F_{T}=C\left(N, v_{F_{T}}\right)$, it also represents the cores of the $T$-face games. Remarkably, Figure 1 contains a lot of information about the geometry of the cores of strictly convex games and, moreover, does it in a noteworthy visual way.

\section{References}

Gillies, D. B. (1953): "Some Theorems on $n$-Person Games," Ph.D. thesis, Princeton.

ICHIISHI, T. (1981): "Super-modularity: applications to convex games and the greedy algorithm for LP," Journal of Economic Theory, 25, 283-286.

Shapley, L. S. (1971): "Cores of Convex Games," International Journal of Game Theory, $1,11-26$. 


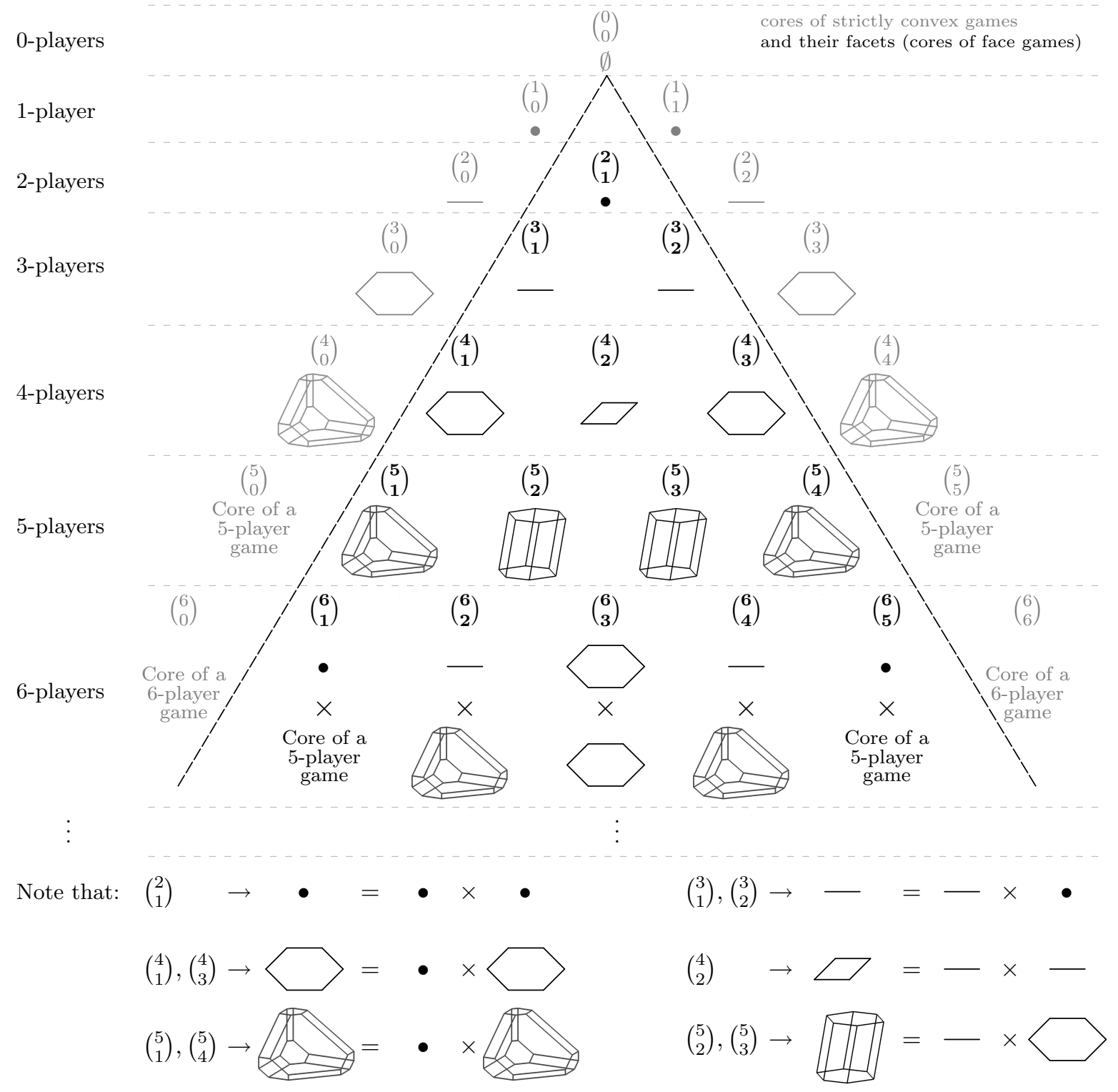

Figure 1: Pascal's triangle and core complexity

- For each $n$, below $\left(\begin{array}{l}n \\ n\end{array}\right)$ and $\left(\begin{array}{l}n \\ 0\end{array}\right)$ we depict the core of an $n$-player strictly convex game. The core of any other $n$-player strictly convex game being combinatorially equivalent to it (Corollary [3).

- For each $n$ and each $t \in\{1, \ldots, n-1\}$, below each $\left(\begin{array}{c}n \\ t\end{array}\right)$ we depict one of the $\left(\begin{array}{c}n \\ t\end{array}\right)$ combinatorially equivalent $T$-facets (with $|T|=t$ ) of $C(N, v)$ (Corollary 2 ).

- For each $n$ and each $t \in\{1, \ldots, n-1\}$, the facets below $\left(\begin{array}{c}n \\ t\end{array}\right)$ and $\left(\begin{array}{c}n \\ n-t\end{array}\right)$ are also combinatorially equivalent to each other (Corollary 2).

- Differently form Pascal's triangle, for each $n$ and each $t \in\{1, \ldots, n-1\}$, the facets below $\left(\begin{array}{c}n \\ t\end{array}\right)$ are not obtained through $\left(\begin{array}{c}n-1 \\ t-1\end{array}\right)$ and $\left(\begin{array}{c}n-1 \\ t\end{array}\right)$. Instead, they are the product of two cores: the one below $\left(\begin{array}{l}t \\ t\end{array}\right)$ and the one below $\left(\begin{array}{l}n-t \\ n-t\end{array}\right)$ (Corollary 1). For $n \leq 5$, this products are represented in the three lines below the triangle.

- Finally, Corollary 4 is readily verified from the picture. 